Alakbar A. Aliyev', Babak G. Abbasov', AhadA. Mammadov ${ }^{3}$

Baku State University ${ }^{1}$, Qafqaz University ${ }^{2}$, Baku, Azerbaijan ${ }^{3}$

DOI: $10.25045 /$ jpit.v07.i2.03

$\underline{\text { aaliyev@mail.ru }}{ }^{1}, \underline{\text { babbasov@qu.edu.az }}{ }^{2}, \underline{\text { ahadm@qu.edu.az }}^{3}$

\title{
COMPARATIVE ANALYSIS OF EFFICIENCY OF AODV, DSR AND WRP ROUTING PROTOCOLS IN AD-HOC NETWORKS
}

Routing protocols yield various outcomes in different situations. Thus far, many scholars investigated existing and recently proposed routing protocols in different environments. Due to increase in the amount of mobile devices and access points, the routing protocols have undergone the performance degradation. The paper analyzes and evaluates three routing protocols based on the node density and node mobility. According to the analysis results, DSR (Dynamic Source Routing) protocol has demonstrated better performance in some cases and values. However, AODV (Ad-hoc On-Demand Distance Vector) routing protocol demonstrated overall better performance than others.

Keywords: Ad-hoc networks, AODV protocol, DSR protocol, WRP protocol, node density parameters.

\section{Introduction}

Ad-hoc network is a group of wireless mobile nodes connected through the cooperation over multi-hop wireless links without the use of fixed infrastructure and centralized control. Some environments, such as the application scope of this type of networks, can be as follows: communication of rescue teams with one another during natural disasters, such as earthquake and flood, without the need for specific network, or exchange of tactical information among the soldiers in a case of war, and/or the environments needed for business/work data exchange among the entrepreneurs during sessions and meetings, and etc. For the delivery of data packets without overloading the network, Adhoc network should have dynamic and accessible speed. However, as Ad-hoc network have high mobility and low speed characteristics, there is a need for routing protocols [1].

Until now, several routing protocols have been proposed for ad-hoc networks [2-4]. The mechanism used by these protocols are generally divided into three groups: table- driven, ondemand and hybrid routing protocols (a combination of the both). The on-demand routing protocols send a routing request only when it is required, the table-driven routing protocols information gather routing information regardless of traffic in all areas of the network. The hybrid routing protocols combine the advantages of both types of protocols. Due to these mechanism differences, Ad-hoc routing protocols have been recently compared according to various efficiency indicators [5-7]. These comparisons consider different situations, environments and scenarios. Obviously, each routing protocol shows various positive and negative results in different situations. Nevertheless, in the time of rapid growth in the number of mobile devices, the demand for the distribution network in devices through HotSpot is also promptly increasing. Behavior of existing protocols was studied through the environment simulation, and various experiments were carried out to solve new problems. In this article, only the analysis of the node density and node intensity is evaluated. According to the analysis results, DSR (Dynamic Source Routing) protocol has demonstrated better performance in some cases and values. However, AODV (Ad-hoc OnDemand Distance Vector) routing protocol demonstrated overall better performance than others.

\section{Routing Protocols}

In general, the Wireless Ad-hoc Network (WANET) routing protocols are divided into three main categories:

- Proactive or table-driven routing protocols;

- Reactive or on-demand routing protocols;

- Hybrid protocols (combination of proactive and reactive protocols). 
Proactive or table-driven routing protocols. Proactive routing protocols store routing information, which is used to connect to other network nodes, as a routing table of each node. In case of changes in the network topology (for example, when existing nodes changes positions, new links are established, or available nodes or links fail), the changes occurred in the links are applied to all nodes in the network. Before the package transmission, the routes of all possible directions are detected by this way and the tables are updated. Whilst MANET (Mobile Ad-hoc Networks) network uses proactive protocol, the overload of the network with control messages by these prompt changes occurred in the network topologies, as well as reduced actual transmission of additionally sent messages are considered of the most critical issues. Proactive protocols are OLSR (Optimized Link State Routing Protocol) [8], DSDV (Destination Sequenced Distance Vector) [9], WRP (Wireless Routing Protocol) [10] and FSR (Fisheye State Routing) [ 11].

Reactive or on-demand routing protocols. In this type of routing protocols, the routes are identified on demand, and maintained by each node of the network. The nodes spend control messages to the network to detect new route. Only when a new route is detected, a bandwidth is used until the end of the process. The advantage of the reactive routing protocols is that little routing data is required in each node. The reason for this is that the acquisition and storage of routing data of all nodes of the network is not required. Another advantage of reactive routing protocols is that the middle or transmitter nodes do not have to decide on the choice of route. One of the major disadvantages is the delays caused by the identification of the route. In addition, the regular route changes occurred on the network and spread of control messages that are characteristic for MANET networks, as well as detection of new routes required for these changes causes the load of the network. Reactive protocols are DSR [12], AODV [13] and ABR (Associativity Based Routing) [14] and etc.

Hybrid protocols (combination of proactive and reactive protocols). Network loading caused by the control messages in reactive protocols and initial delays caused by the detection of routing makes obstacles for these protocols to be the most optimal solution for MANET networks. On the other hand, as it is required to constantly set large-scale routing table on large networks, the utilization of proactive protocols is not an acceptable solution. Therefore, the use of a protocol, combining the advantages of both types of protocols on MANET networks, is considered the most effective solution. This type of protocols may include ZRP (Zone Routing Protocol) protocol [15]. However, it should be noted that this protocol cannot eliminate all the shortcomings.

In this paper, we have selected two major protocols: AODV on-demand routing protocol and DSR table-driven WRP routing protocol in order to implement comparative analysis of the efficiency of the routing protocols.

DSR protocol. DSR protocol is absolutely on-demand Ad-hoc network protocol, and its routing process consists of two parts: Route Discovery and Route Maintenance [12]. When one node is sending data to any other node and when there is no any available route in the same direction in the Route Cache, it enables the process of discovering the source route. Discovering the target and a unique identifier, the source sends Route Request (RREQ) [16]. When the node receives RREQ request it cancels the request, if the request is received for the first time, the node adds its address to the list of the request and spreads it widely on the network again (Figure 1a). When RREQ request is delivered to the target, the target sends Route Reply (RREP) to the source. The reply includes the addresses of all the nodes obtained by the route based on the requests (Figure 1b). When Route Reply is delivered to the source, it adds a new route to its own route cache. The process of route service, which is a part of routing process, includes this stage [12]. Figure 1a provides the process of route discovery between the node No1 (source) and the node No12 (target). Figure 1b provides the process of sending Route Reply in the opposite direction from the target to the source node. 


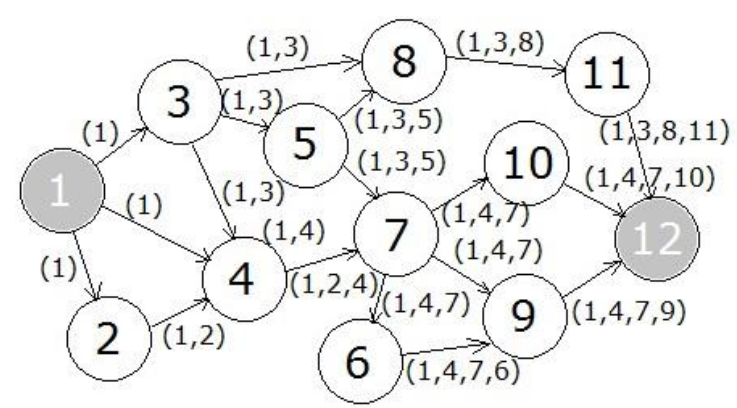

(a)

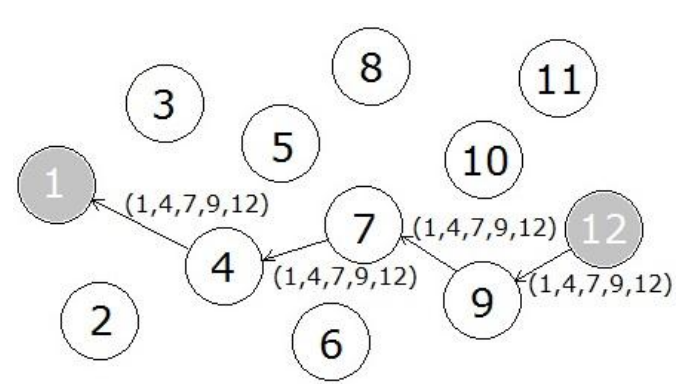

(b)

Figure 1. The process of routing discovery of DSR protocol: a) the process of route discovery

(RREQ); b) sending Route Reply (RREP) to the source

After revealing the route, the source node posts a full list of nodes from the source to the target at the message header. Each node, along the route, passes the message to the next hop, and tries to approve whether the message reached the node or not. If the response delivery fails, the message is resent. If the response delivery fails once more, transmitter node sends route error to the source of the message after discovering the broken link to the next node. After that, the source node deletes the broken link from route cache. The source node selects and uses one of the other routes from its cache in order to send the next messages in the same direction, if necessary, it enables the process of a new route discovery.

$A O D V$ protocol. AODV protocol is one of the reactive routing protocols, which loads the network less, and can adapt quickly to the dynamic changes of the links on wireless and mobile ad-hoc networks. In AODV protocol, the processes of route discovery and service are not enabled, unless it is required to establish contact between the two nodes. The protocol uses the destination sequence number in order to guarantee the release of the new routing loops [13]. As in DSR protocol, AODV routing protocol implements two processes: route discovery and route service. When one node is sending a message to another and when there is no any routing data in the same destination, the process of route discovery is enabled and extended Route Reply is sent to all neighboring nodes. When the device receiving the request gets a new route, the route sends the reply; otherwise, the hop count passes the route request to its neighboring nodes by increasing its value by a unit (Figure 2). If the source receives route error message as the result of link error, it enables the process of route discovery again, in order to get a new by the destination [13].
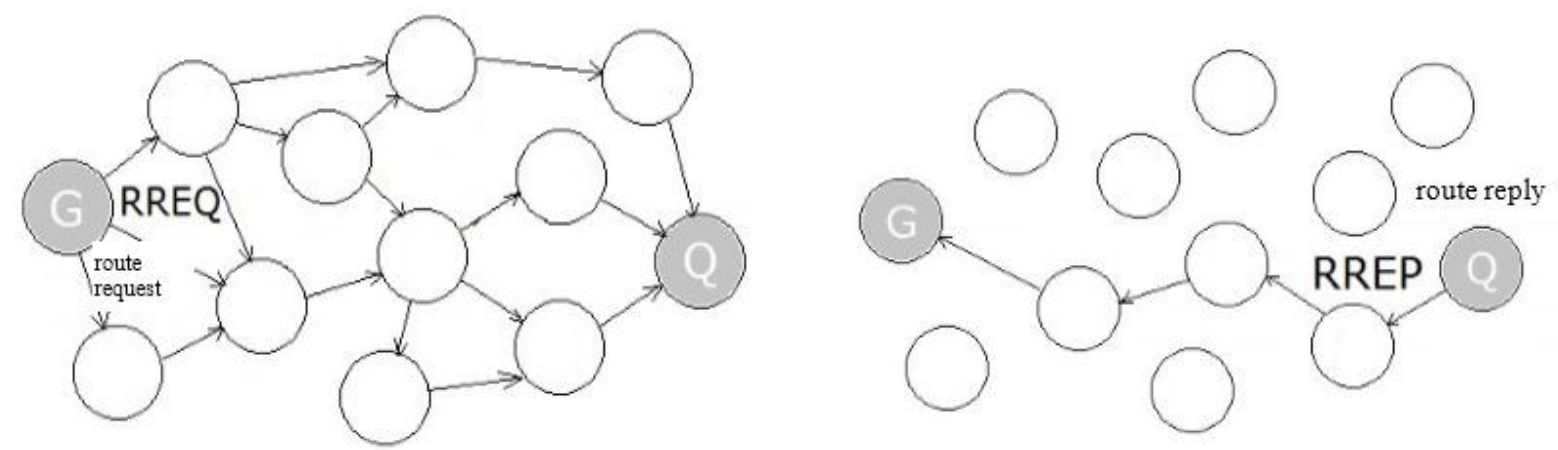

Figure 2. Route discovery of AODV protocol

WRP protocol. WRP protocol is a pro-active routing protocol used for mobile Ad-hoc networks (MANET). The protocol uses an updated version of distance-vector routing protocol, which uses Bellman-Ford algorithm to find routes [10]. On MANET networks, WRP protocol uses specific mechanisms to ensure reliable data exchange and to reduce the route loops occurred in the 
nodes due to the mobility. In the route table, the routes in all directions are located together with the next hop and cost metric. To reduce the route value, the route is determined using neighboring hop. There are different methods to calculate the value of a link. After being assigned, the values are usually kept in a separate table. In the case of the link changes on the network, the update messages include only the most recent changes instead of full route schedule. If updated messages to be sent are not available, empty HELLO messages are permanently sent to the network with periodic intervals to keep the links updated [10]. On small-sized networks, WRP protocol involves a few routing tables, and as the result, the protocols adapting more promptly than others are observed. However, if multiple tables are used, the services to be provided become complicated, which require ultimately more memory and computing power. Therefore, on very large and dynamic Ad-hoc wireless networks, WRP protocol performs fairly low efficiency.

\section{Conducted experiments}

Simulation experiments are conducted to compare the effectiveness of abovementioned three routing protocols. In this section, experimental modeling, analysis, and the results of the simulation experiments are provided.

GloMoSim (Global Mobile Information System Simulator) simulators are used to compare the routing protocols examined in this paper. GloMoSim software is one of the most optimal and free of charge simulation tools used for large-scale wired and wireless networks [17]. The study is conducted through GloMoSim, and the efficiency comparisons are evaluated by the parameters of three routing protocols as end-to-end delay, packet delivery rate and throughput the parameters. During the experiment, these parameters are determined as follows:

End-to-end delay. This value is a mean time measure from the beginning of the message sent by the source (including the route acquisition delays) to its reception by the target. The measure includes processing, queueing and propagation delays. When the value of the end-to-end delay is low, a better efficiency is achieved:

$$
\text { Mean } U U G=\frac{1}{N} \sum_{n=1}^{N}\left(T Q_{n}-T G_{n}\right)
$$

Here, $U U G$ denotes the end-to-end delay, $\mathrm{TG}_{n}$ - sending date of the $n$-th message, $\mathrm{TQ}_{\mathrm{n}}$ receiving date of the $n$-th message, $N$ - the number of received data packets.

Package delivery rate. This setting is the ratio of the number of packets successfully delivered to the target with the total number of packets sent by the source. This value shows the maximum throughput capacity achieved by the network. Therefore, the high rate of packet delivery on the networks is very desirable:

\section{$P D R=(\Sigma$ received the CBR packages $/ \Sigma$ sent packages $C B R) * 100$}

Throughput capacity of the network. This option is a total number of packets, which are successfully delivered to separate destinations, and calculated as the ratio of delivered packages with the simulation period $(T s)$ of 8 -fold of the package volume:

Throughput $=($ the number of delivered packets $*$ package volume $* 8) / T$ s.

In simulation experiments, the control options as node density (experiment 1) and node mobility (experiment 2) are used. Then, above-mentioned measures as the end-to-end delay, packet delivery rate and throughput are measured and evaluaded.

The movement directions of the nodes are modeled using random waypoint model. Each node in the model remains constant throughout the pause time. When the process ends, the node starts moving within the network terrain in arbitrarily predefined direction. Once the node reaches new destination point, it stands motionless again during the pause time. As soon as the time expires, the node starts moving again in another arbitrary direction. This action continues until the end of the simulation process. 
The size of the network, where the experiments are carried out, is $2 \mathrm{~km}^{2}(2000 \times 2000 \mathrm{~m})$, the width of all transition lines is 2 Mbps and simulation period is set 900 seconds (Table 1). In both experiments, seven arbitrary sources and target node pair are used. Each of the randomly selected seven sources sends CBR (Constant Bit Rate) packages to the randomly seven targets. The volume of the packages is set at 512-byte.

In the simulation, TwoRay is selected as a distribution model for the physical level protocol, IEEE 802.11 standard - for MAC level protocol, and "radio-accnoise" model - for sending and receiving the packets. All arrangements used in the simulation experiments are given in Table 1.

Experimental design. In simulation, two different experiences on node density (experiment 1) and node mobility (experiment 2) is carried out. Details of the experiments are as follows:

$>$ The first experiment measures the impact of the node density on the end-to-end delay, packet delivery rate and throughput. The impact of node density is examined with all the nodes moving in two different frequencies within the network terrain with the pause time of 30 seconds. Separately, 10, 20, 30, 40 and 50 nodes are used and studied, the first of which is moving in $0 \mathrm{~m} / \mathrm{s}$ (inactive), and the second in $40 \mathrm{~m} / \mathrm{s}$.

$>$ The second experiment, as opposed to others, only 10 nodes are used and the impact of the node mobility is examined. In this experiment, as in experiment 1 , movement directions of the nodes are defined by Random waypoint model. With the pause time of 30 seconds, the speed of nodes are measured in six different phases: very high speed (50 $\mathrm{m} / \mathrm{s})$, high speed $(40 \mathrm{~m} / \mathrm{s})$, medium speed $(30 \mathrm{~m} / \mathrm{s})$, low speed $(20 \mathrm{~m} / \mathrm{s})$, very low speed $(10 \mathrm{~m} / \mathrm{s})$, and inactive $(0 \mathrm{~m} / \mathrm{s})$.

In both experiments, the same ways and random sampling methods are used. In addition, in order to achieve the accuracy of the test results, the simulation is carried out hundred times for each configuration and the mean value is chosen. Moreover, in some cases, extremely upper and lower values are not taken into account.

Table 1

Simulation Settings

\begin{tabular}{|l|c|}
\hline \multicolumn{1}{|c|}{ Settings } & Values \\
\hline Simulator & GloMoSim v2.03 \\
\hline Information package & TWO-RAY $\mathrm{TH}$ \\
\hline propagation model & RADIO-ACCNOISE \\
\hline Package sharing model & Random Waypoint \\
\hline Mobility model & IEEE 802.11 \\
\hline MAC level & IP (Internet Protocol v4) \\
\hline Network protocol & AODV, DSR and WRP \\
\hline Routing protocol & $0,10,20,30,40,50 \mathrm{~m} / \mathrm{s}$ \\
\hline Mobility speed & $10,20,30,40,50$ \\
\hline Number of nodes & 512 bytes \\
\hline Packet volume & 30 seconds \\
\hline Pause time & $2000 \mathrm{~m} \times 2000 \mathrm{~m}$ \\
\hline Simulation area & 900 seconds \\
\hline Simulation duration &
\end{tabular}

Experiment results and analyses. First, node density experiment is carried out with two different speeds - inactivity and a maximum of $40 \mathrm{~m} / \mathrm{s}$. The average delay results are provided in Figure 3. The results obtained in the case of inactivity, in AODV protocol, if only 40 and 50 nodes are used, unlike the other two protocols, mean delay value varies within 0.3 and 0.2 . In all other 
cases, delay values of all three protocols vary within 0 and 0.05 . In the first experiment, proactive routing protocol WRP performs the best result (Figure 3a). Compared to reactive protocols, DSR protocol performs better result than AODV protocol. However, when the nodes move with the maximum speed of $40 \mathrm{~m} / \mathrm{s}$, DSR protocol performs excessively high delay (Figure $3 \mathrm{~b}$ ).

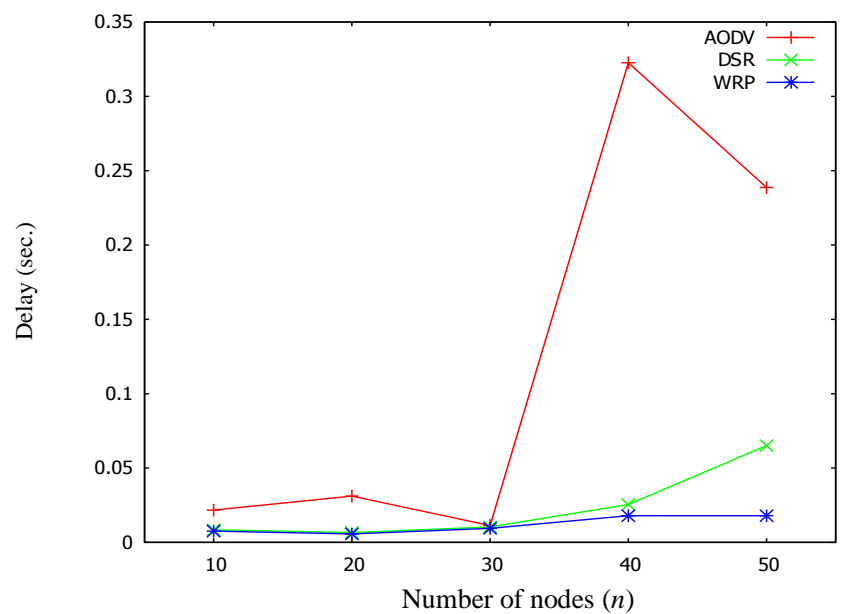

a)

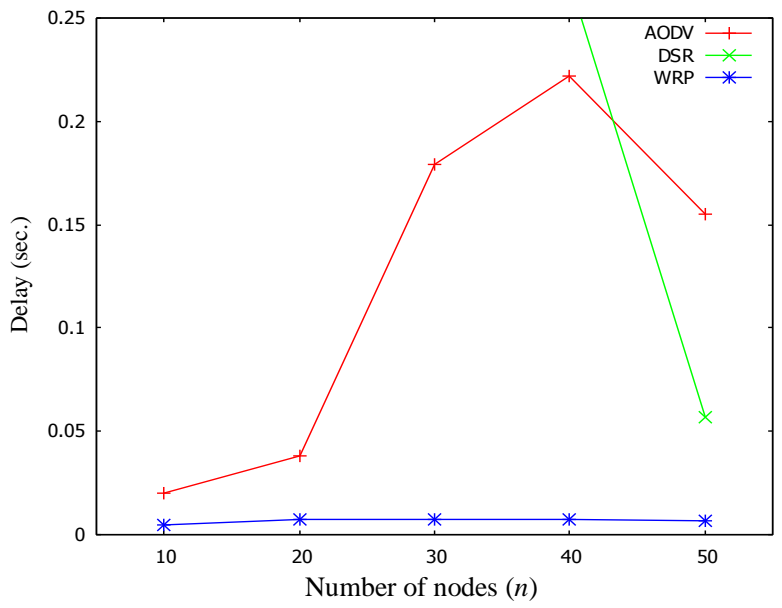

b)

Figure 3. Average delay results: a) at the speed of $0 \mathrm{~m} / \mathrm{s}$; b) at the speed of $40 \mathrm{~m} / \mathrm{s}$

According to these experiments, WRP performs the best result again, and AODV, unlike in the case of inactivity, performs better results compared to DSR protocol. DSR leaves behind AODV by 0.1 seconds only when 50 nodes are moving.

In the second phase of the first experiment, comparison is conducted by the packet delivery rate. As seen from the results shown in Figure 4, in the case of inactivity, when using all three routing protocols, all the nodes performed similar results (Figure 4a). However, when the nodes move at the speed of $40 \mathrm{~m} / \mathrm{s}$, the results dramatically changed (Figure $4 \mathrm{~b}$ ).

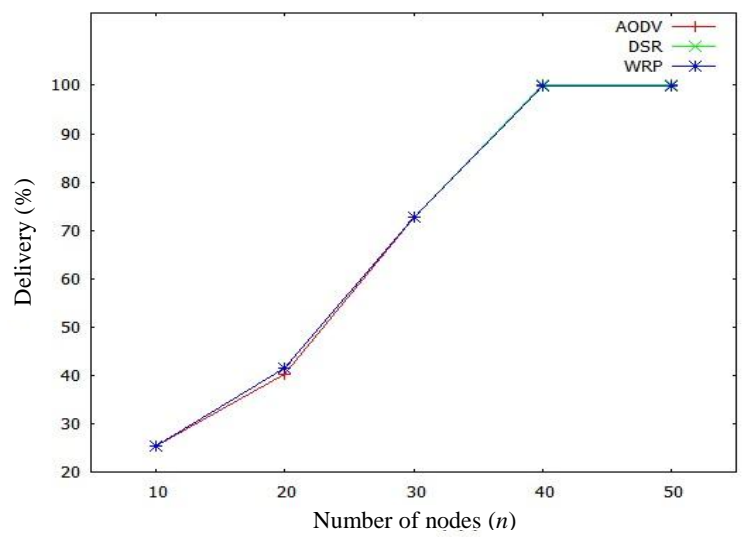

a)

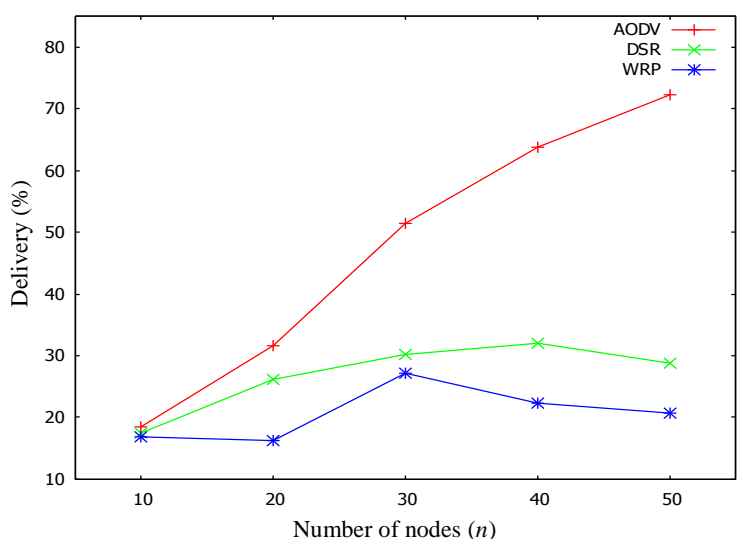

b)

Figure 4. Mean packet delivery rate: a) at the speed of $0 \mathrm{~m} / \mathrm{s}$; b) at the speed of $40 \mathrm{~m} / \mathrm{s}$

As it is seen from the figure, WRP performs averagely $20 \%$ result, DSR - 27\%, and AODV $47 \%$. As the number of nodes increases, AODV performs better results compared to other protocols. For example, when 50 nodes are moving, packet delivery rate of AODV protocol is $72.22 \%$ (Figure 4b). 
The last phase of the experiment is carried out on throughput. As shown in Figure 5a, in the case of inactivity, the routing protocols showed similar results. However, it should be particularly noted that, when the number of nodes is 30 , the highest throughput is $1637 \mathrm{bps}$. When the nodes are moving with 30 second intervals (pause time) and at the speed of $40 \mathrm{~m} / \mathrm{s}$, all three protocols showed different results (Figure 5b).

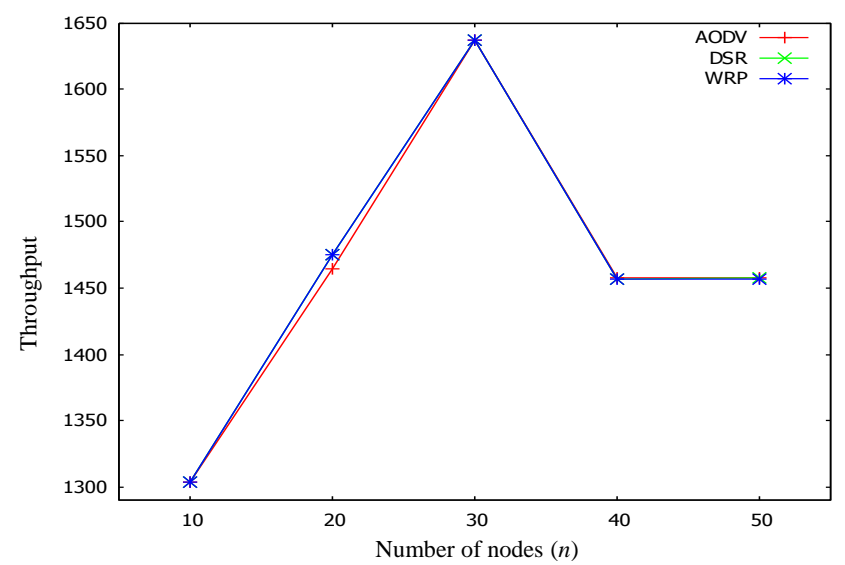

a)

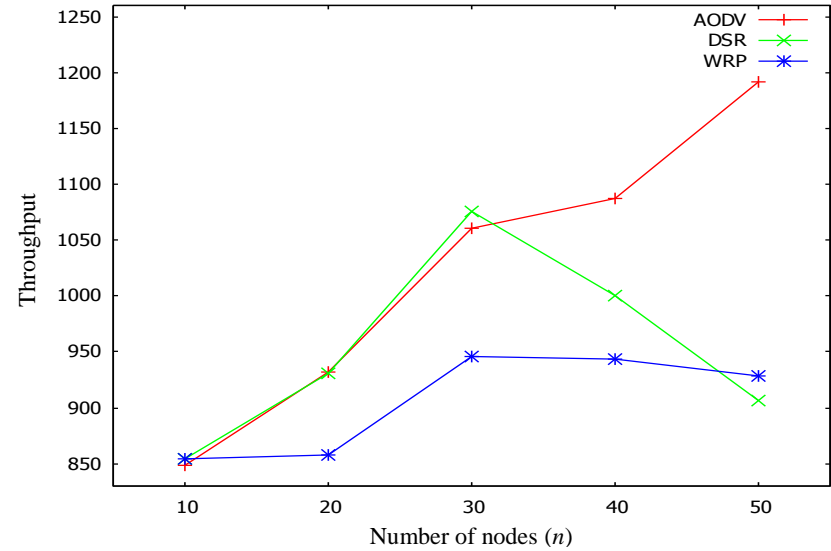

b)

Figure 5. Mean throughput: a) at the speed of $0 \mathrm{~m} / \mathrm{s}$; b) at the speed of $40 \mathrm{~m} / \mathrm{s}$

In the second experiment, only 10 nodes are used to measure the node mobility, which are moving at six different speeds. Delay coefficient for WRP and AODV protocols is 0.01, whereas for the DSR protocol, it is increased to 12 seconds. Looking at Figure 6, DSR protocol clearly seems to be a very slow depending on its mobility. Nevertheless, when the research is conducted according to the packet delivery rate and throughput, at some speeds, DSR protocol performs better results than others (Figure 7).

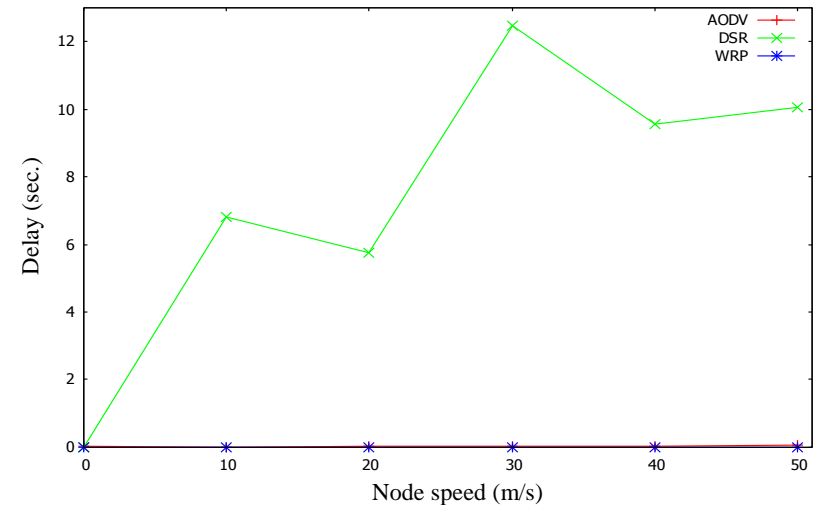

Figure 6. Mean delay with the use of 10 nodes

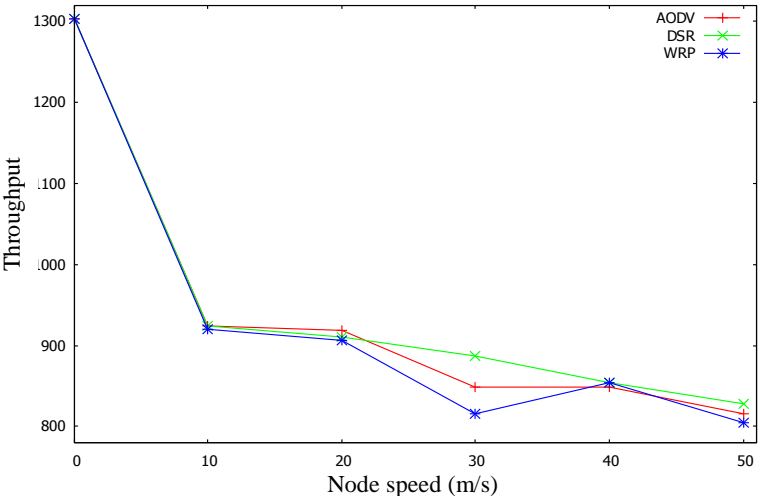

Figure 7. Mean throughput value with the use of 10 nodes 
As it is seen from the figures, although, the best packet delivery rate is performed by AODV protocol (Figure 8), when 10 nodes are moving at the speed of 30, 40 and $50 \mathrm{~m} / \mathrm{s}$, DSR performs better results than the others for their throughput capacity. When moving at a speed of 20 meters per second, AODV protocol, and each three protocols at the stability and moving at the speed of $10 \mathrm{~m} / \mathrm{s}$ performs the same result.

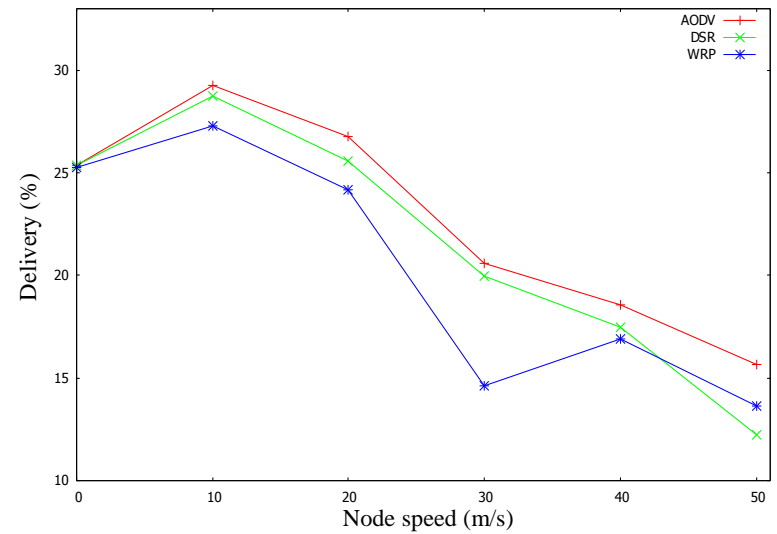

Figure 8. Mean packet delivery rate with the use of 10 nodes

\section{Conclusion and future research}

In this study, simulation experiments are conducted among AODV, DSR and WRP routing protocols using GloMoSim software, and their efficiency results are compared. The protocols' efficiency comparisons are evaluated by the parameters of three routing protocols as the end-to-end delay, packet delivery rate and throughput parameters. In the simulation experiments, the control options, as node density (experiment 1) and node mobility (experiment 2), are used, and derived values are measured and evaluated. Experience shows that, although, in the case of inactivity and at a maximum speed of $40 \mathrm{~m} / \mathrm{s}$, proactive routing protocol WRP performs the best result for the node density, as the number of nodes increases, AODV delivers the best results compared to other protocols for the packet delivery rate. In the first experiment, conducted on the throughput capacity, WRP the protocol, unlike previous experiences, performs the worst result regardless of the number of nodes. In addition, the DSR protocol shows higher throughput rate when using 10, 20 and 30 nodes, and AODV protocol, when using 40 and 50 nodes. The second experiment measured the mobility of nodes, in which DSR protocol is clearly found to be very slow, depending on the mobility characteristics. However, when the research is conducted according to packet delivery rate and throughput capacity, at some speeds, DSR protocol shows better rates than others. When it comes to the overall results, the best result is performed by AODV routing protocol.

Looking at the results of the study, we draw the conclusion that the use of new methods and techniques are required for AODV protocol to show the best results in all cases of the experiments. To this end, in the next phase, it is expected to fully examine AODV protocol, to improve algorithm and methods, and to offer a new approach. As a result of this new approach, AODV protocol is expected to perform the best result in all experiences of this study.

\section{References}

1. Chlamtac I., Conti M., Liu J. Mobile ad hoc networking: imperatives and challenges, Ad Hoc Networks 1, 2003, pp.13-64.

2. Royer E.M., Toh C.-K. A Review of Current Routing Protocols for Ad Hoc Mobile Wireless Networks, IEEE Personal Communications, 1999, vol.6, no.2, pp.46-55.

3. Jayakumar G., Gopinath G. Ad Hoc Mobile Wireless Networks Routing Protocols - A Review, Journal of Computer Science, 2007, vol.3, no.8, pp.574-582. 
4. Radwan A.A.A., Mahmoud T.M., Houssein E.H. Evaluation comparison of some ad hoc networks routing protocols, Egyptian Informatics Journal, 2011, vol.12, no.2, pp.95-106.

5. Das S.R., Perkins C.E., Royer E.M. Performance Comparison of Two On-demand Routing Protocols for Ad Hoc Networks, IEEE InfoCom, 2000, vol.1, pp.3-12.

6. Dyer T.D., Boppana R.V. A Comparison of TCP Performance over Three Routing Protocols for Mobile Ad Hoc Networks, ACM Symposium on Mobile Ad Hoc Networking \& Computing (MobiHoc), 2001, pp.56-66.

7. Raju J., Garcia-Luna-Aceves J.J. A Comparison of On-Demand and Table Driven Routing for Ad-hoc Wireless Networks, IEEE ICC '00, 2000, pp.1702-1706.

8. Jacquet P., Muhlethaler P., Clausen T., Laouiti A., Qayyum A., Viennot L. Optimized link state routing protocol for AdHoc networks, IEEE Multi Topic Conference, 2001, pp.62-68.

9. Murthy S., Garcia-Luna-Aceves J.J. Loop-free internet routing using hierarchical routing trees, IEEE INFOCOM, 1997, vol.1, pp.101-108.

10. Murthy S., Garcia-Luna J.J. An efficient routing protocol for wireless networks, ACM Mobile Networks and Applications Journal, 1996, pp.183-197.

11. Iwata A., Chiang C.C., Pei G., Gerla M., Chen T.W. Scalable routing strategies for ad hoc wireless networks, IEEE Journal of Selected Areas on Communications, 1999, vol.17, no.8, pp.1369-1379.

12. Johnson D. B., Maltz D. A. Dynamic Source Routing in Ad Hoc Wireless Networks, Mobile Computing, Kluwer Academic Publisher, 1996, ch.5, pp.153-181

13. Perkins C.E., Royer E. Ad-hoc On-Demand Distance Vector Routing, 2nd IEEE Workshop on Mobile Computing \& Systems and Applications, 1999, pp.90-100.

14. Toh Chai-K. Associativity-Based Routing for Ad-hoc Mobile Networks, Wireless Personal Communication Journal, 1997, vol.4, no.2, pp.103-139

15. Peralman M.R., Haas Z.J.Determining the optimal configuration for the zone routing protocol, IEEE Journal on Selected Areas in Communications, 1999, vol.17, no.8, pp.1395-1414.

16. Johnson B.D., Maltz A.D., Broch J. A Self-stabilizing Link-Cluster Algorithm in Mobile Ad Hoc Networks,8th International Symposium on Parallel Architectures, Algorithms, and Networks, 2005, pp. 436-441.

17. Rajaj L., et.al. GloMoSim: A Scalable Network Simulation Environment, Technical Report, No. 990027, Computer Science Dept., UCLA, 1999. 